\title{
Thermodynamic integration via differential evolution: A method for approximating marginal likelihoods
}

\author{
Nathan J. Evans ${ }^{a}$ and Jeffrey Annis ${ }^{b}$ \\ ${ }^{a}$ Department of Psychology, University of Amsterdam, The Netherlands \\ ${ }^{b}$ Department of Psychology, Vanderbilt University, USA
}

Word count:

5,290

Keywords: marginal likelihood approximation — Bayes factor - Bayesian model selection

- cognitive modeling (JA)

Correspondence may be addressed to: nathan.j.evans@uon.edu.au (NJE); jeff.annis@vanderbilt.edu 


\begin{abstract}
A typical goal in cognitive psychology is to select the model that provides the best explanation of the observed behavioral data. The Bayes factor provides a principled approach for making these selections, though the integral required to calculate the marginal likelihood for each model is intractable for most cognitive models. In these cases, Monte Carlo techniques must be used to approximate the marginal likelihood, such as thermodynamic integration (TI; Friel \& Pettitt, 2008; Lartillot \& Philippe, 2006), which relies on sampling from the posterior at different powers (called power posteriors). TI can become computationally expensive when using population Markov chain Monte Carlo (MCMC) approaches such as differential evolution MCMC (DE-MCMC; Turner, Sederberg, Brown, \& Steyvers, 2013) that require several interacting chains per power posterior. Here, we propose a method called thermodynamic integration via differential evolution (TIDE), which aims to reduce the computational burden associated with TI by using a single chain per power posterior. We show that when applied to non-hierarchical models, TIDE produces an approximation of the marginal likelihood that closely matches TI. When extended to hierarchical models, we find that certain assumptions about the dependence between the individual and group level parameters samples (i.e., dependent/independent) have sizable effects on the TI approximated marginal likelihood. We propose two possible extensions of TIDE to hierarchical models, which closely match the marginal likelihoods obtained through TI with dependent/independent sampling in many, but not all, situations. Based on these findings, we believe that TIDE provides a promising method for estimating marginal likelihoods, though future research should focus on a detailed comparison between the methods of approximating marginal likelihoods for cognitive models.
\end{abstract}


When creating and testing psychological models, the goal is often to select the model among a pool of models that provides the best explanation of the observed data (Roberts \& Pashler, 2000). Recent advancements in computing technology has led to an increasing number of formalized models (e.g., Ratcliff, 1978; Brown \& Heathcote, 2008), which can produce precise quantitative predictions. The advantage of the precise, quantitative predictions of formalized models also comes with an additional challenge: How do we quantitatively choose the model that provides the best explanation of the psychological process? Although this choice may seem like an easy one, where the model that provides the best fit to the observed data should be selected, models that have a greater flexibility will often "over-fit" to the noise within the sample, leading to poor generalization (Myung \& Pitt, 1997; Myung, 2000; Roberts \& Pashler, 2000). Making this choice between models is a process known as model selection (Myung \& Pitt, 1997). Traditional model selection methods such as AIC (Akaike, 1974) or BIC (Schwarz et al., 1978) combine a goodness-of-fit statistic with a penalty term for model flexibility based on the number of parameters in the model. Although these methods are computationally simple, they can be inadequate in situations where, for example, parameters affect model flexibility differently (Myung \& Pitt, 1997). In this article, we will use the Bayesian approach to model selection, an approach that balances goodness-of-fit and model flexibility in a consistent framework (Myung \& Pitt, 1997; Shiffrin, Lee, Kim, \& Wagenmakers, 2008).

The Bayesian approach to model selection is most easily introduced by first discussing the more familiar Bayesian approach to parameter estimation. The goal of Bayesian parameter estimation is to find the joint posterior distribution of the parameters, $\boldsymbol{\theta}, p(\boldsymbol{\theta} \mid \boldsymbol{D}, \mathcal{M})$, where $\mathcal{M}$ is the model, and $\boldsymbol{D}$ is the data vector. The joint posterior distribution is given by Bayes' rule:

$$
p(\boldsymbol{\theta} \mid \boldsymbol{D}, \mathcal{M})=\frac{p(\boldsymbol{D} \mid \boldsymbol{\theta}, \mathcal{M}) p(\boldsymbol{\theta} \mid \mathcal{M})}{p(\boldsymbol{D} \mid \mathcal{M})},
$$


where $p(\boldsymbol{D} \mid \boldsymbol{\theta}, \mathcal{M})$ is the likelihood function, $p(\boldsymbol{\theta} \mid \mathcal{M})$ is the prior probability of the parameters, and $p(\boldsymbol{D} \mid \mathcal{M})$ is the marginal likelihood found by marginalizing over all possible parameter values.

While Bayesian parameter estimation is primarily concerned with estimating the posterior distribution, $p(\boldsymbol{\theta} \mid \boldsymbol{D}, \mathcal{M})$, the quantity of interest in Bayesian model selection is the marginal likelihood:

$$
p(\boldsymbol{D} \mid \mathcal{M})=\int p(\boldsymbol{D} \mid \boldsymbol{\theta}) p(\boldsymbol{\theta} \mid \mathcal{M}) d \boldsymbol{\theta}
$$

The marginal likelihood can be used to perform Bayesian model selection by obtaining the posterior odds ratio:

$$
\frac{p\left(\mathcal{M}_{1} \mid \boldsymbol{D}\right)}{p\left(\mathcal{M}_{2} \mid \boldsymbol{D}\right)}=\frac{p\left(\boldsymbol{D} \mid \mathcal{M}_{1}\right)}{p\left(\boldsymbol{D} \mid \mathcal{M}_{2}\right)} \times \frac{p\left(\mathcal{M}_{1}\right)}{p\left(\mathcal{M}_{2}\right)}
$$

where the term $\frac{p\left(\boldsymbol{D} \mid \mathcal{M}_{1}\right)}{p\left(\boldsymbol{D} \mid \mathcal{M}_{2}\right)}$ is the Bayes factor and $\frac{p\left(\mathcal{M}_{1}\right)}{p\left(\mathcal{M}_{2}\right)}$ is the prior model odds. Bayesian model selection is often performed in the absence of the prior model odds. In this case, only the Bayes factor must be computed, a measure of evidence provided by the data in favor of one model over the other, which accounts for model flexibility by integrating over all possible parameter values (Myung \& Pitt, 1997).

For very simple models with only a few parameters, sometimes the marginal likelihood integral can be analytically solved or estimated via standard numerical integration techniques. In many cases, where the model has more than a few parameters and the integral is intractable, we must resort to Monte Carlo techniques. The Monte Carlo approach relies on the relationship between certain forms of integrals and their corresponding representations as expected values, which can be approximated via sampling techniques (Evans \& Brown, 2018).

One of the most straightforward Monte Carlo estimators of the marginal likelihood is the arithmetic mean estimator (Kass \& Raftery, 1995; Evans \& Brown, 2018), in which the 
average likelihood under samples from the prior is used as an approximation to the marginal likelihood. Although the arithmetic mean estimator is both conceptually simple and easy to implement, a large number of samples (e.g., 10,000,000+) is often required to obtain an accurate approximation of the marginal likelihood in a complex cognitive models (e.g., the Linear Ballistic Accumulator; LBA; Brown \& Heathcote, 2008) with around 6 parameters, and the number of samples required continues to increase with dimensionality (Evans \& Brown, 2018). Thus, the arithmetic mean estimator is impractical for many complex cognitive models on standard computing hardware. Instead, Graphical Processing Units (GPUs) capable of drawing a large number of samples in parallel can be used to obtain accurate estimates (Evans \& Brown, 2018). Alternatively, there are a host of methods that can be used to approximate the Bayes factor or marginal likelihood using standard computing hardware such as bridge sampling (Gronau et al., 2017), the Savage-Dickey method (Wagenmakers, Lodewyckx, Kuriyal, \& Grasman, 2010), Chib's method (Chib, 1995), or the product method (Lodewyckx et al., 2011). Note, the focus of this article will not be on comparing methods for marginal likelihood estimation. For those interested in a comparison of methods, we refer the reader to reviews by Friel and Wyse (2012) and Liu et al. (2016).

The present article will focus on improving an existing method known as as thermodynamic integration (TI; Lartillot \& Philippe, 2006; Friel \& Pettitt, 2008). In TI, the posterior distribution is raised to powers between 0 and 1. Samples are drawn from each of these power posteriors and are then used to calculate the marginal likelihood. TI can be computationally expensive because sampling must be done over many power posteriors. This is especially true when using population Markov chain Monte Carlo (MCMC) techniques, such as differential evolution MCMC (DE-MCMC; Ter Braak, 2006; Turner et al., 2013), to sample from the power posteriors as they require several chains for each power 
posterior. The DE-MCMC approach has become popular due to its ability to efficiently sample from models with correlated parameters (see Evans \& Brown, 2017; Evans, Rae, Bushmakin, Rubin, \& Brown, 2017; Evans, Steyvers, \& Brown, 2018 for some applications) by using a set of interacting chains, usually 2-3 times number of individual-level parameters. Thus, DE-MCMC can be computationally burdensome when used in the context of TI, especially as the number of individual-level parameters grows.

Here, we present a variation of TI utilizing DE-MCMC in which only a single chain per power posterior is needed. Our method, which we call TIDE, implements TI within a population MCMC framework, an approach first introduced by Calderhead and Girolami (2009). We found that TIDE provided an approximation of the marginal likelihood that closely matched TI for models with a single subjects. However, when extending the models hierarchically, we found that certain assumptions about the dependence between the individual and group level parameter samples resulted in large differences in the TI approximated marginal likelihood, where the standard dependent sampling results in higher marginal likelihoods than the recently implemented (e.g., Heathcote et al., 2018; implemented within their $D M C$ package) independent sampling. We extended TIDE to these two different situations, with dependent sampling only requiring a natural extension of TIDE, and independent sampling adding the use of past iterations in a manner similar to "Z updating" from an extension of DE-MCMC, DE-MCz (ter Braak \& Vrugt, 2008). We refer to the latter, independent sampling extension as TIDEz, and find that both TIDE and TIDEz can closely match to the marginal likelihood obtained through TI in some situations, but that this doesn't occur in all situations. However, when making inferences in our empirical data example, we find that both methods and sampling assumptions result in the same general inferences.

The remainder of this article will take the following format. First, we will discuss 
the TI method, and why TI can become computationally burdensome in some situations. Second, we will explain how integrating TI and DE-MCMC to form our new method, TIDE, can lead to a reduction in the computational burden associated with TI. Third, we present extensions of TIDE to hierarchical models, and show that they closely agree with the marginal likelihoods obtained by TI in some situations, using both simulated and empirical data.

\section{Thermodynamic Integration}

Thermodynamic Integration (TI) (Friel \& Pettitt, 2008; Lartillot \& Philippe, 2006) is a method for estimating the marginal likelihood of a model. TI defines a set of posterior distributions. The likelihood of each posterior is raised to a power, $t_{j}=\{0, \ldots, 1\}$ (called the temperature). These new posteriors are referred to as power posteriors and are defined as:

$$
p\left(\boldsymbol{\theta} \mid \boldsymbol{D}, t_{j}\right)=\frac{p(\boldsymbol{D} \mid \boldsymbol{\theta})^{t_{j}} p(\boldsymbol{\theta})}{\int p(\boldsymbol{D} \mid \boldsymbol{\theta})^{t_{j}} p(\boldsymbol{\theta}) d \boldsymbol{\theta}},
$$

where $j=\{1, \ldots, k\}$ (called the temperature rung) indexes each of the $k$ temperatures, dropping the model notation, $\mathcal{M}$, for brevity. The power posterior with a temperature of 0 is the prior distribution, and the power posterior with a temperature of 1 is the posterior distribution. After obtaining samples from each power posterior, $\boldsymbol{\theta}_{i, j} \sim p\left(\boldsymbol{\theta} \mid \boldsymbol{D}, t_{j}\right)$, the average log-likelihoods, $\frac{1}{n} \sum_{i=1}^{n} \ln p\left(\boldsymbol{D} \mid \boldsymbol{\theta}_{i, j}\right)$, are computed. These form $k$ points along a one-dimensional curve with respect to $t$, and the area under this curve is an estimate of the marginal likelihood. Since it is a one-dimensional curve, its area is easily estimated with standard numerical integration techniques. Friel and Pettitt (2008) suggest the trapezoidal rule: 


$$
p(\boldsymbol{D}) \approx \sum_{j=2}^{k} \frac{t_{j}-t_{j-1}}{2}\left[\frac{1}{n} \sum_{i=1}^{n} \ln p\left(\boldsymbol{D} \mid \boldsymbol{\theta}_{i, j}\right)+\frac{1}{n} \sum_{i=1}^{n} \ln p\left(\boldsymbol{D} \mid \boldsymbol{\theta}_{i, j-1}\right)\right] .
$$

TI relies on the discretization of temperatures, referred to as the temperature schedule. A temperature schedule in which $t_{j}$ is set to the $(j-1)^{t h}$ quantile of a $\operatorname{Beta}(\alpha, 1)$ distribution, where $\alpha=.3$ has been shown to work well (Xie, Lewis, Fan, Kuo, \& Chen, 2010; Friel \& Pettitt, 2008):

$$
t_{j}=\left(\frac{j-1}{k-1}\right)^{\frac{1}{\alpha}}
$$

where $k$ the total number of temperatures, $j=\{1, \ldots, k\}$. More about TI and its implementation can be found in Annis, Evans, Miller, \& Palmeri, 2018, and the exact mathematical details of TI can be found in the Appendix.

Notice that TI suffers from two major sources of error, the discretization of temperatures, and MCMC error. Discretization error can be reduced by increasing the number of temperature rungs, and MCMC error can be reduced by increasing the number MCMC samples per temperature rung. Although error reduction in TI is straightforward, increasing the number of MCMC samples and temperature rungs leads to increased computational workload.

Thus, a major drawback of the method is the computational burden that TI can impose in order to obtain accurate marginal likelihood estimates. For example, in prior work (Annis et al., 2018), we found the number of temperatures needed to obtain a stable estimate of the marginal likelihood to be around 20-35 for hierarchical LBA models. This computational burden increases when a population MCMC algorithm is used to obtain samples, where several interacting chains are necessary for each power posterior. The population MCMC approach we focus on is differential evolution MCMC (DE-MCMC; 
Ter Braak, 2006; Turner et al., 2013), which has become popular in cognitive psychology due to its ability to efficiently sample from posteriors with correlated parameters. It requires a number of chains equal to 2-3 times the number of parameters in the largest updating block, which within hierarchical models is commonly the number of parameters per individual. In the next section, we propose a method based on DE-MCMC that aims to reduce the number of chains needed for each power posterior to one.

\section{Thermodynamic Integration via Differential Evolution (TIDE)}

The algorithm we present combines TI and DE-MCMC so that only a single chain needs to be run for each power posterior, which provides the benefits of DE-MCMC while avoiding the costly overhead of having to run several chains per power posterior. The algorithm relies on the same approach as Calderhead and Girolami (2009) who originally proposed implementing TI within a population MCMC framework. Here, we propose an extension we refer to as thermodynamic integration via differential evolution (TIDE).

DE-MCMC uses the genetic algorithm, differential evolution, to generate proposals. Generally, genetic algorithms are a class of algorithms that involve some amount of crossover between different existing elements to create new elements, as well as some possible random mutations that change the new elements. DE-MCMC uses a set of interacting chains that are all simultaneously sampling from the posterior. To create a new proposal on iteration $i$ for chain $c, \boldsymbol{\theta}_{i, c}$, the previous value on that chain, $\boldsymbol{\theta}_{i-1, c}$, is added to the difference between two randomly chosen other chains, $l$ and $m$ (i.e., a crossover):

$$
\boldsymbol{\theta}_{i, c}=\boldsymbol{\theta}_{i-1, c}+\gamma\left(\boldsymbol{\theta}_{i-1, l}-\boldsymbol{\theta}_{i-1, m}\right)+\epsilon
$$

where $\gamma$ controls the size of "jump" for the new proposal, and $\epsilon$ is a small amount of random noise (i.e., a mutation). The proposal is then accepted or rejected according to the 
Metropolis Hastings step, and the process continues on to create a proposal for the next chain. DE-MCMC forms a population of interacting chains, $\boldsymbol{\Theta}=\left(\boldsymbol{\theta}_{1}, . ., \boldsymbol{\theta}_{C}\right)$, where $C$ is the total number of chains, with the following product distribution:

$$
p(\boldsymbol{\Theta} \mid \boldsymbol{D})=\frac{1}{\prod_{c=1}^{C} \int p\left(\boldsymbol{D} \mid \boldsymbol{\theta}_{c}\right) p\left(\boldsymbol{\theta}_{c}\right) d \boldsymbol{\theta}} \prod_{c=1}^{C} p\left(\boldsymbol{D} \mid \boldsymbol{\theta}_{c}\right) p\left(\boldsymbol{\theta}_{c}\right)
$$

where $\boldsymbol{\theta}_{i, c} \sim p(\boldsymbol{\theta} \mid \boldsymbol{D})$. This is the typical case in which samples are drawn from the posterior distribution. It is also possible to sample from a power posterior at temperature, $t_{j}$. In this case, the population of chains forms the following product distribution:

$$
p\left(\boldsymbol{\Theta} \mid \boldsymbol{D}, t_{j}\right)=\frac{1}{\prod_{c=1}^{C} \int p\left(\boldsymbol{D} \mid \boldsymbol{\theta}_{c}\right)^{t_{j}} p\left(\boldsymbol{\theta}_{c}\right) d \boldsymbol{\theta}} \prod_{c=1}^{C} p\left(\boldsymbol{D} \mid \boldsymbol{\theta}_{c}\right)^{t_{j}} p\left(\boldsymbol{\theta}_{c}\right),
$$

where $\boldsymbol{\theta}_{i, c} \sim p\left(\boldsymbol{\theta} \mid \boldsymbol{D}, t_{j}\right)$. This is what we refer to as standard TI with DE-MCMC or just standard TI. A drawback of this approach is that it requires $C$ chains for each power posterior. To more elegantly combine DE-MCMC with TI, TIDE forms a population of interacting chains, $\boldsymbol{\Theta}=\left(\boldsymbol{\theta}_{1}, . ., \boldsymbol{\theta}_{k}\right)$, with following product distribution:

$$
p(\boldsymbol{\Theta} \mid \boldsymbol{D}, \boldsymbol{t})=\frac{1}{\prod_{j=1}^{k} \int p\left(\boldsymbol{D} \mid \boldsymbol{\theta}_{j}\right)^{t_{j}} p\left(\boldsymbol{\theta}_{j}\right) d \boldsymbol{\theta}} \prod_{j=1}^{k} p\left(\boldsymbol{D} \mid \boldsymbol{\theta}_{j}\right)^{t_{j}} p\left(\boldsymbol{\theta}_{j}\right)
$$

where $\boldsymbol{\theta}_{i, j} \sim p\left(\boldsymbol{\theta} \mid \boldsymbol{D}, t_{j}\right)$. Thus, TIDE only requires a single chain per power posterior by allowing chains to interact between power posteriors instead of only within power posteriors. After sampling, the average log-likelihood under each chain is computed, $\frac{1}{n} \sum_{i=1}^{n} \ln p\left(\boldsymbol{D} \mid \boldsymbol{\theta}_{i, j}\right)$, and the trapezoidal rule is used to estimate the marginal likelihood. It should also be noted that TIDE is equally applicable to any other method that approximates the marginal likelihood using power posteriors, such as TI "corrected", or steppingstone sampling (SS; see Annis et al., 2018 for a tutorial on both of these methods). 
Next, we compare the performance of TIDE to standard TI for the data of an individual simulated subject, and extend it to hierarchical models for groups of subjects, and compare its performance to standard TI for a group of simulated subjects.

Algorithm 1 displays pseudo-code for how to implement TIDE. Once starting points for the parameter values (i.e., $\boldsymbol{\theta}_{1}$ ) are obtained, an iterative process is performed to obtain the posterior samples (for $i \leftarrow 2$ to $n$ do), which is performed for each temperature (for $j \leftarrow 1$ to $k$ do). Each iteration for each temperature requires selecting two other random chains (i.e., two other temperatures), creating the DE proposal using those selected chains, and then deciding whether to accept the proposal based upon the Metropolis Hastings step. After the iterative process is complete, the marginal likelihood estimate is obtained using the trapezoidal rule. 


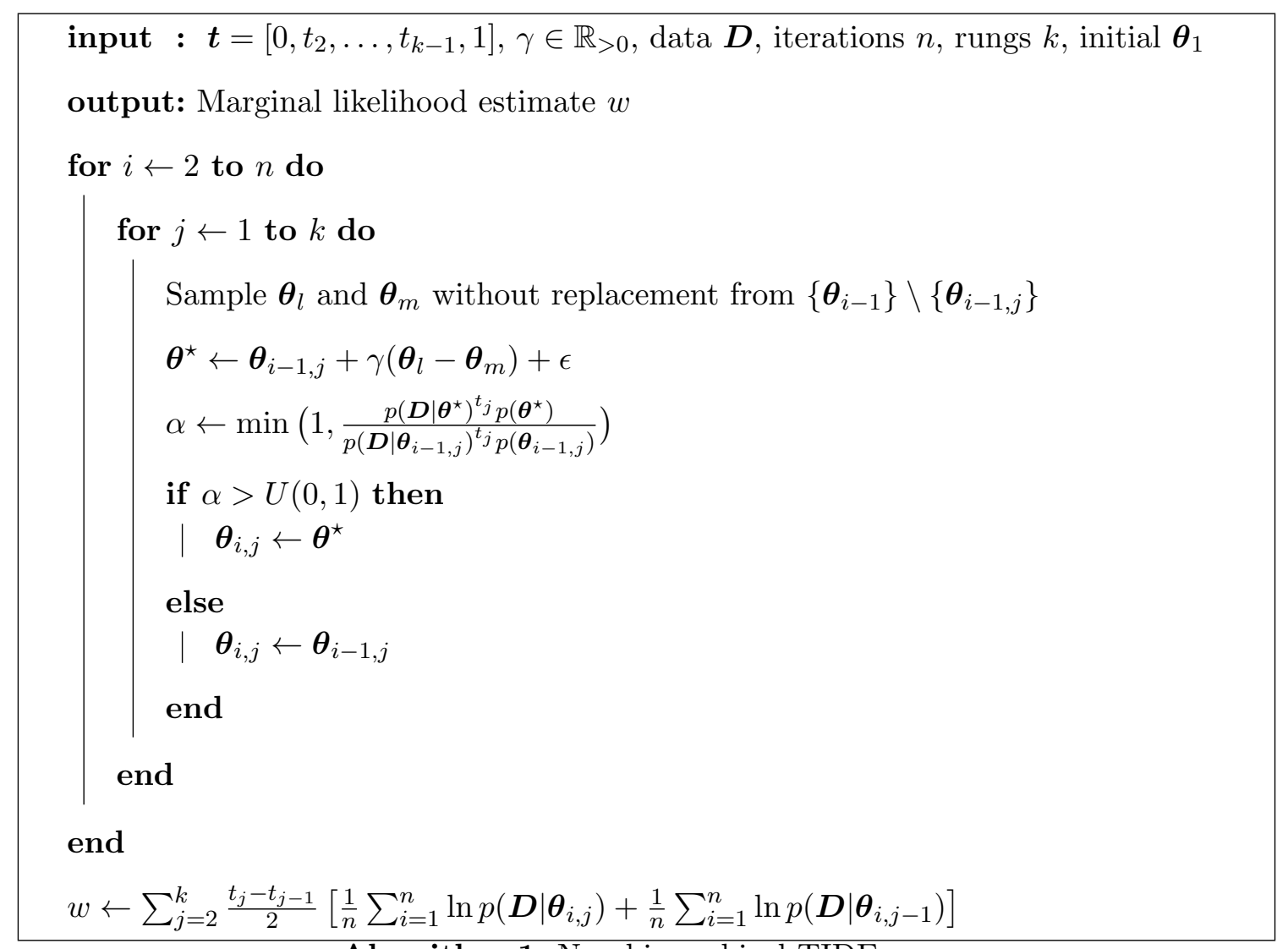

Algorithm 1: Non-hierarchical TIDE

TIDE for Hierarchical models

Hierarchical models have become an increasingly popular method within cognitive psychology for making inferences on groups of participants (Shiffrin et al., 2008). Hierarchical models involve estimating parameters for individual participants (i.e., the individuallevel, $\boldsymbol{\theta})$, and constraining the estimates of each parameter for all individuals to follow a group-level distribution of the parameters (i.e., the group-level, $\phi$ ). Hierarchical models provide key benefits over non-hierarchical estimation, allowing information from different individuals to constrain the estimation of one another (commonly known as shrinkage), and providing a method of performing group-level inference on the entire dataset from experiments. Formally, hierarchical models can be defined as: 


$$
\begin{aligned}
\boldsymbol{D}_{p} & \sim p\left(\boldsymbol{D}_{p} \mid \boldsymbol{\theta}_{p}\right) \\
\boldsymbol{\theta}_{p} & \sim p\left(\boldsymbol{\theta}_{p} \mid \boldsymbol{\phi}\right) \\
\boldsymbol{\phi} & \sim p(\boldsymbol{\phi}),
\end{aligned}
$$

where $p$ indexes the participant. For hierarchical models of this form, the power posterior is given by:

$$
p\left(\boldsymbol{\theta}, \boldsymbol{\phi} \mid \boldsymbol{D}, t_{j}\right)=\frac{p(\boldsymbol{D} \mid \boldsymbol{\theta}, \boldsymbol{\phi})^{t_{j}} p(\boldsymbol{\theta}, \boldsymbol{\phi})}{\iint p(\boldsymbol{D} \mid \boldsymbol{\theta}, \boldsymbol{\phi})^{t_{j}} p(\boldsymbol{\theta}, \boldsymbol{\phi}) d \boldsymbol{\theta} d \boldsymbol{\phi}} .
$$

TIDE now forms two populations of interacting chains, $\boldsymbol{\Theta}=\left(\boldsymbol{\theta}_{1}, . ., \boldsymbol{\theta}_{k}\right)$ and $\boldsymbol{\Phi}=$ $\left(\phi_{1}, . ., \phi_{k}\right)$, with the following product distribution:

$$
p(\boldsymbol{\Theta}, \boldsymbol{\Phi} \mid \boldsymbol{D}, \boldsymbol{t})=\frac{1}{\prod_{j=1}^{k} \iint p\left(\boldsymbol{D} \mid \boldsymbol{\theta}_{j}\right)^{t_{j}} p\left(\boldsymbol{\theta}_{j}, \boldsymbol{\phi}_{j}\right) d \boldsymbol{\theta} d \boldsymbol{\phi}} \prod_{j=1}^{k} p\left(\boldsymbol{D} \mid \boldsymbol{\theta}_{j}\right)^{t_{j}} p\left(\boldsymbol{\theta}_{j}, \boldsymbol{\phi}_{j}\right)
$$

where $\left(\boldsymbol{\theta}_{i, j}, \boldsymbol{\phi}_{i, j}\right) \sim p\left(\boldsymbol{\theta}, \boldsymbol{\phi} \mid \boldsymbol{D}, t_{j}\right)$. After drawing samples from the joint distribution, the individual-level samples are used to compute the average likelihoods in the same way as before, $\frac{1}{n} \sum_{i=1}^{n} \ln p\left(\boldsymbol{D} \mid \boldsymbol{\theta}_{i, j}\right)$, which are in turn are used in the trapezoidal rule to obtain an estimate of the marginal likelihood. Although samples are drawn from the joint distribution, $p\left(\boldsymbol{\theta}, \boldsymbol{\phi} \mid \boldsymbol{D}, t_{j}\right)$, only the individual-level samples, $\boldsymbol{\theta}_{i, j}$, are needed in the computation of marginal likelihood estimate. Thus, the group-level priors only enter indirectly into the estimation of the marginal likelihood by constraining the $\boldsymbol{\theta}_{i, j}$ samples. A proof is given in the Appendix.

Algorithm 2 displays pseudo-code for how to implement hierarchical TIDE. The algorithm is very similar to the non-hierarchical TIDE in Algorithm 1, though the itera- 
tive process now involves two stages: updating the group-level parameters, and updating the individual-level parameters. The first stage, updating the group-level parameters, is similar to updating the parameters in non-hierarchical TIDE, and requires selecting two other random chains (i.e., two other temperatures), creating the DE proposal using those selected chains, and then deciding whether to accept the proposal based upon the Metropolis Hastings step. Note that the Metropolis Hastings step shown here does not involve the probability of the data under the individual-level parameters (i.e., $p(\boldsymbol{D} \mid \boldsymbol{\theta})$ ), as the proposal does not involve new individual-level parameters, and therefore, the terms involving identical individual-level parameters cancel out in the Metropolis Hastings step. The second stage, updating the individual-level parameters, involves a loop over participants (for $p \leftarrow 1$ to $P$ do), as the parameters for each individual are updated separately to reduce dimensionality. After this, the process involves the same steps as stage one, though for the individual-level parameters for this participant. Also note that the Metropolis Hastings step shown here does not involve the prior probability of the group-level parameters (i.e., $p(\phi))$, for the same canceling out reasons as above. 


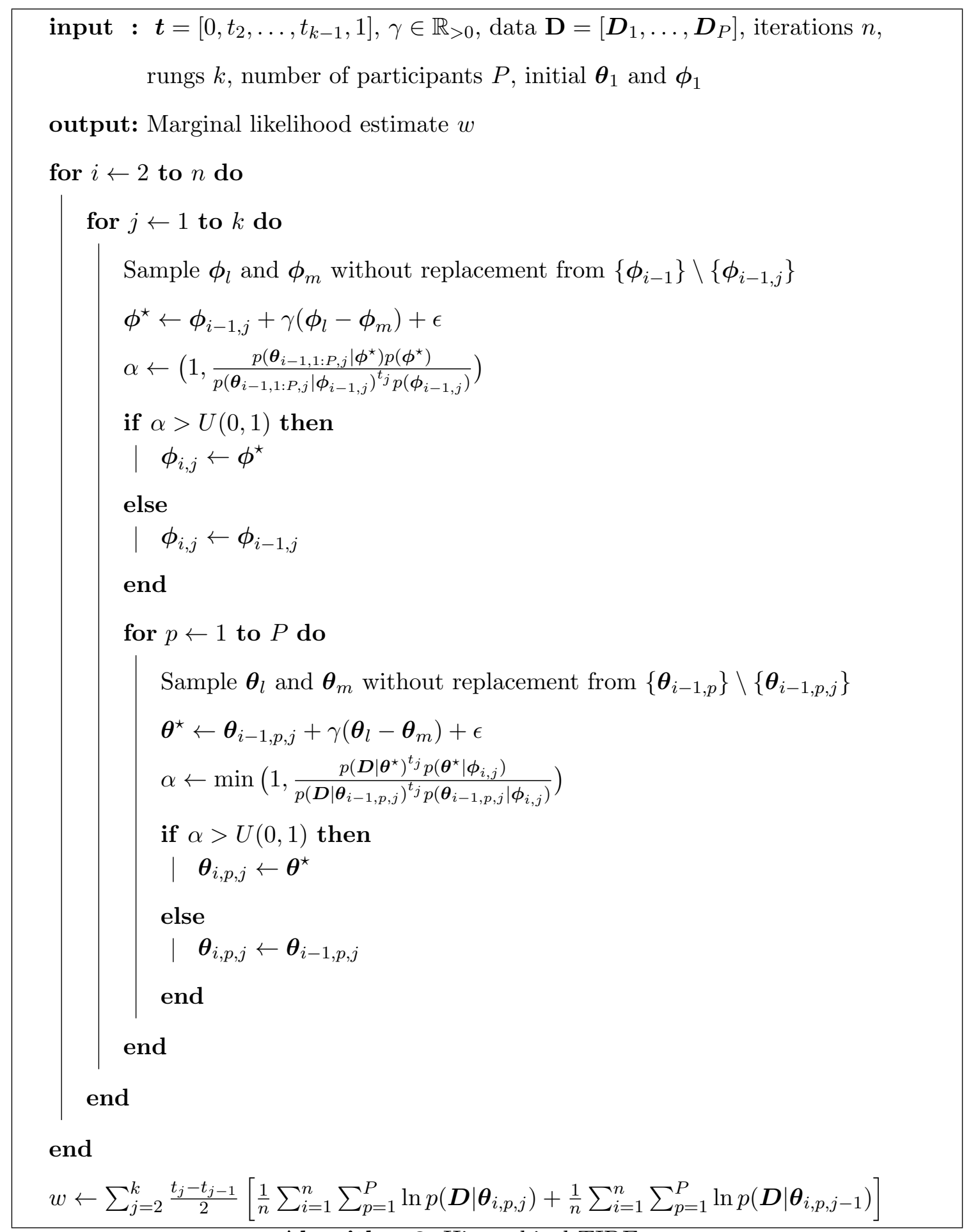

Algorithm 2: Hierarchical TIDE 


\section{Thermodynamic Integration via Differential Evolution with $Z$ updating (TIDEz)}

Although hierarchical models are conceptually simple to implement, practical difficulties have been reported when extending cognitive models to these hierarchical structures. For example, when hierarchically estimating the diffusion model (Ratcliff, 1978), some researchers have recommended fixing specific parameters to only be estimated at the group level (i.e., all individuals share the same parameter value), due to these parameters having only small influences on the model likelihood, which results in difficulties accurately estimating the full hierarchical structure (e.g., Wiecki, Sofer, \& Frank, 2013; the grouplevel fixing of inter-trial variability parameter is implemented in their $H D D M$ package). A related problem has been referred to as the "zero-variance trap", where all participants values for a certain parameter - a parameter that only has a small effect on the likelihood, and therefore, can be highly influenced by the group-level constraints - converge to a single value, resulting in the group-level variability between participants approaching zero, and the values becoming "stuck". However, when using the DE-MCMC framework and a specific system of "blocking" outlined below, a simple solution can be used to remedy this problem by "breaking the dependency" between the individual and group-level parameters (Tillman, Osth, van Ravenzwaaij, \& Heathcote, 2017; Heathcote et al., 2018).

When using the DE-MCMC algorithm, the parameters being updated at any one point are usually split into different sampling blocks, as large numbers of parameters (i.e., high dimensionality) can lead to inefficient sampling. These blocks involve only specific parameters having proposals generated, and the posterior likelihood being conditioned on the other parameters. Commonly, a different block is used for the parameters of each participant, and for the group-level parameters. This means that the parameters of each individual participant are updated according to: 


$$
p\left(\boldsymbol{\theta}_{i, p} \mid \boldsymbol{\phi}_{i}, \boldsymbol{D}\right) \propto p\left(\boldsymbol{D} \mid \boldsymbol{\theta}_{i}\right) p\left(\boldsymbol{\theta}_{i} \mid \boldsymbol{\phi}_{i}\right)
$$

where $p$ indexes the participant and $i$ indexes the current sample. The parameters of the group are updated according to:

$$
p\left(\boldsymbol{\phi}_{i} \mid \boldsymbol{\theta}_{i}, \boldsymbol{D}\right) \propto p\left(\boldsymbol{\theta}_{i} \mid \boldsymbol{\phi}_{i}\right) p\left(\boldsymbol{\phi}_{i}\right)
$$

Importantly, this system of blocking allows for a simple method of "breaking the dependence" between the individual and group level parameters, which has been found to remedy some of the practical issues that can occur in hierarchical models discussed above (Tillman et al., 2017; Heathcote et al., 2018). Specifically, the method involves randomly pairing the values of the $\boldsymbol{\theta}$ and $\boldsymbol{\phi}$ parameters of different chains for the purposes of updating: for the updating of the $\boldsymbol{\theta}$ parameters for a specific chain, the $\boldsymbol{\phi}$ parameters from another random chain is selected to be conditioned on, and vise versa ${ }^{1}$. Equation 14 can then be changed to:

$$
p\left(\boldsymbol{\theta}_{i, p} \mid \boldsymbol{\phi}_{l}, \boldsymbol{D}\right) \propto p\left(\boldsymbol{D} \mid \boldsymbol{\theta}_{i}\right) p\left(\boldsymbol{\theta}_{i} \mid \boldsymbol{\phi}_{l}\right),
$$

where $i$ indexes the current sample (i.e., the current chain), and $l$ indexes another random chain. Likewise, Equation 15 can then be changed to:

$$
p\left(\boldsymbol{\phi}_{i} \mid \boldsymbol{\theta}_{l}, \boldsymbol{D}\right) \propto p\left(\boldsymbol{\theta}_{l} \mid \boldsymbol{\phi}_{i}\right) p\left(\boldsymbol{\phi}_{i}\right)
$$

Essentially, this random pairing "breaks the dependence" between the individual and group-level parameters, resulting in the joint posterior having independent samples of individual and group-level parameters, as opposed to the standard sampling method, which

\footnotetext{
${ }^{1}$ As far as we are aware, this solution was first suggested by Brandon M. Turner.
} 
contains a full joint posterior. It should also be noted that the random pairing is performed as sampling without replacement: that this, each individual-level chain is randomly paired with one other group-level chain. However, this simple solution is no longer possible with TIDE, as each chain estimates the power posterior for a different temperature, and therefore, a different target distribution.

Although we cannot sample across chains, we can sample across time. This solution is similar to an updating procedure called Z-updating in DE-MCz (ter Braak \& Vrugt, 2008) and so we refer to this algorithm as TIDEz. As discussed previously, the $\boldsymbol{\theta}$ and $\boldsymbol{\phi}$ parameters are updated separately, in different blocks. TIDEz randomly pairs the $\boldsymbol{\theta}$ and $\phi$ samples using previous posterior samples. Equation 14 can then be changed to:

$$
p\left(\boldsymbol{\theta}_{i, p} \mid \boldsymbol{\phi}_{z}, \boldsymbol{D}_{p}\right) \propto p\left(\boldsymbol{D}_{p} \mid \boldsymbol{\theta}_{i}\right) p\left(\boldsymbol{\theta}_{i} \mid \boldsymbol{\phi}_{z}\right)
$$

where $i$ indexes the current sample, and $z$ indexes a random previous posterior sample. Likewise, Equation 15 can then be changed to:

$$
p\left(\boldsymbol{\phi}_{i} \mid \boldsymbol{\theta}_{z}, \boldsymbol{D}\right) \propto p\left(\boldsymbol{\theta}_{z} \mid \boldsymbol{\phi}_{i}\right) p\left(\boldsymbol{\phi}_{i}\right)
$$

Note that we only use previous posterior samples after a certain number of initial iterations (i.e., not immediately, when the parameters may be a long way from the posterior), and we only reach a certain maximum number of iterations into the past. This introduces two extra "tuning" parameters that need to be set for the TIDEz algorithm: When the Z update starts ("zStart"), and the maximum number of iterations that can be reached into from the past ( " $z L a g$ "). In our applications here, we set $z$ Start to 2,000 , and $z$ Lag to 250.

Algorithm 3 displays pseudo-code for how to implement hierarchical TIDEz. The algorithm is almost identical to the hierarchical TIDE in Algorithm 2, with a two key ex- 
ceptions. Firstly, the iterative process shown here starts at zStart, as iterations before this work in an identical manner to hierarchical TIDE. Secondly, in the line before the creation of the DE proposal, the previous iteration of the individual-level/group-level parameter to pair with the current group-level/individual-level update is chosen, based on the $z L a g$ value. 
input : $\boldsymbol{t}=\left[0, t_{2}, \ldots, t_{k-1}, 1\right], \gamma \in \mathbb{R}_{>0}$, data $\mathbf{D}=\left[\boldsymbol{D}_{1}, \ldots, \boldsymbol{D}_{P}\right]$, iterations $n$, rungs $k$, number of participants $P$, Z-update iteration $z$ Start, initial $\boldsymbol{\theta}_{1:(z \operatorname{Start}-1)}$, and $\boldsymbol{\phi}_{1:(z \text { Start }-1)}$

output: Marginal likelihood estimate $w$

for $i \leftarrow z$ Start to $n$ do

for $j \leftarrow 1$ to $k$ do

Sample $\phi_{l}$ and $\phi_{m}$ without replacement from $\left\{\phi_{i-1}\right\} \backslash\left\{\phi_{i-1, j}\right\}$

Sample $\boldsymbol{\theta}_{z, 1: P, j}$ from $\left\{\boldsymbol{\theta}_{(i-z L a g):(i-1), 1: P, j}\right\}$

$\boldsymbol{\phi}^{\star} \leftarrow \boldsymbol{\phi}_{i-1, j}+\gamma\left(\phi_{l}-\boldsymbol{\phi}_{m}\right)+\epsilon$

$\alpha \leftarrow \min \left(1, \frac{p\left(\boldsymbol{\theta}_{z, 1: P, j} \mid \boldsymbol{\phi}^{\star}\right)^{t} j p\left(\boldsymbol{\phi}^{\star}\right)}{p\left(\boldsymbol{\theta}_{z, 1: P, j} \mid \phi_{i-1, j}\right)^{t_{j}} p\left(\phi_{i-1, j}\right)}\right)$

if $\alpha>U(0,1)$ then

$\phi_{i, j} \leftarrow \phi^{\star}$

else

$\phi_{i, j} \leftarrow \phi_{i-1, j}$

end

for $p \leftarrow 1$ to $P$ do

Sample $\boldsymbol{\theta}_{l}$ and $\boldsymbol{\theta}_{m}$ without replacement from $\left\{\boldsymbol{\theta}_{i-1, p}\right\} \backslash\left\{\boldsymbol{\theta}_{i-1, p, j}\right\}$

Sample $\boldsymbol{\phi}_{z, j}$ from $\left\{\boldsymbol{\phi}_{(i-z \operatorname{Lag}):(i-1), j}\right\}$

$\boldsymbol{\theta}^{\star} \leftarrow \boldsymbol{\theta}_{i-1, p, j}+\gamma\left(\boldsymbol{\theta}_{l}-\boldsymbol{\theta}_{m}\right)+\epsilon$

$\alpha \leftarrow \min \left(1, \frac{p\left(\boldsymbol{D} \mid \boldsymbol{\theta}^{\star}\right)^{t_{j}} p\left(\boldsymbol{\theta}^{\star} \mid \boldsymbol{\phi}_{z, j}\right)}{p\left(\boldsymbol{D} \mid \boldsymbol{\theta}_{i-1, p, j}\right)^{t_{j}} p\left(\boldsymbol{\theta}_{i-1, p, j} \mid \boldsymbol{\phi}_{z, j}\right)}\right)$

if $\alpha>U(0,1)$ then

| $\boldsymbol{\theta}_{i, p, j} \leftarrow \boldsymbol{\theta}^{\star}$

else

| $\boldsymbol{\theta}_{i, p, j} \leftarrow \boldsymbol{\theta}_{i-1, p, j}$

end

end

end

end

$w \leftarrow \sum_{j=2}^{k} \frac{t_{j}-t_{j-1}}{2}\left[\frac{1}{n} \sum_{i=1}^{n} \sum_{p=1}^{P} \ln p\left(\boldsymbol{D} \mid \boldsymbol{\theta}_{i, p, j}\right)+\frac{1}{n} \sum_{i=1}^{n} \sum_{p=1}^{P} \ln p\left(\boldsymbol{D} \mid \boldsymbol{\theta}_{i, p, j-1}\right)\right]$ 
Examples

\section{Individual subjects}

Here, we use TIDE to approximate the marginal likelihood for a cognitive model. Specifically, we apply TIDE to the Linear Ballistic Accumulator (LBA; Brown \& Heathcote, 2008), a commonly used model of decision-making (Evans et al., 2017; Ho et al., 2014; Brown, Marley, Donkin, \& Heathcote, 2008; Evans et al., 2018). We use the LBA as a running example because it has an analytically tractable likelihood function, making computation of TIDE quick enough to allow for estimation to be performed within a short time-frame, and variances across independent estimates to be obtained. In addition, the LBA has been the applied model used in previous manuscripts on estimating marginal likelihoods (Evans \& Brown, 2018; Annis et al., 2018), allowing a clear comparison to previous research. We begin by briefly explaining the LBA, before assessing TIDE in the case of data from an individual subject.

The LBA is a model of decision-making that falls within a class of models known as evidence accumulation models (Stone, 1960; Ratcliff, 1978; Ratcliff \& Rouder, 1998; Brown \& Heathcote, 2008). Evidence accumulation models propose that decision-making is the result of the accumulation of evidence for the different choice alternatives until the evidence for one alternative reaches a threshold and a decision is triggered. Specifically, the LBA proposes that this accumulation process involves independent racing accumulators for each alternative, with the rate of evidence accumulation being constant within a decision, but differing between decisions according to a normal distribution, truncated at 0 . Evidence is also proposed to start at a random point for each accumulator that differs between decisions, with the starting evidence being uniformly distributed between 0 and some point less than the decision threshold. Lastly, the model contains some amount of time dedicated to processes outside of the decision, such as perception and motor responding. 
This results in the model having 5 parameters: the mean rate of evidence accumulation over decisions $(v)$, the standard deviation of evidence accumulation over decisions $(s)$, the threshold amount of evidence required to make a decision (b), the upper bound of the uniform distribution of starting evidence $(A)$, and the time dedicated to non-decision related components $(t 0)$.

Specifically, we used the same simulated dataset as Evans and Brown (2018) and Annis et al. (2018). This dataset had two "within-subjects conditions" simulated, with a generating process that had no parameters differing between the conditions, which we call a "simple" dataset. As with Evans and Brown (2018) and Annis et al. (2018), we fit two models to each of these datasets: a "simple" model that constrained all parameters to take the same values over conditions, and a "complex" model that allowed mean drift rate, threshold, and non-decision time to vary over conditions. The specific model definition for data generation and fitting can be found in the Appendix.

The resulting log-marginal likelihood estimates for TIDE (solid lines) can be seen in Figure 1 for the simple data set and Figure 2 for the complex data set. We compare these to the estimates obtained by standard TI (dashed lines), which used 10 chains and 500 iterations per power posterior with a burnin of 1500 iterations. Points are means based on 10 independent replications and error bars are standard deviations. Each panel of the plot corresponds to the marginal likelihood estimate obtained with a given numbers of temperature rungs $(10,20,35$, and 50$)$. The $\mathrm{x}$-axis provides the number of samples taken from the power-posterior of each temperature, and the y-axis is the estimated log-marginal likelihood. When using 35 rungs, TIDE produces log-marginal likelihoods that are close to those obtained through standard TI and have low variance estimates $(\mathrm{SD}<1)$ after 3000 to 3500 samples. When using 50 rungs, this is achieved in roughly 2000 iterations. In addition, when using a small number of rungs (i.e., 10/20), TIDE more closely matches 
the marginal likelihood obtained using a higher number of rungs than standard TI. Thus, our results suggest TIDE may be an efficient method of performing TI when estimating marginal likelihoods for models of individual subjects. 


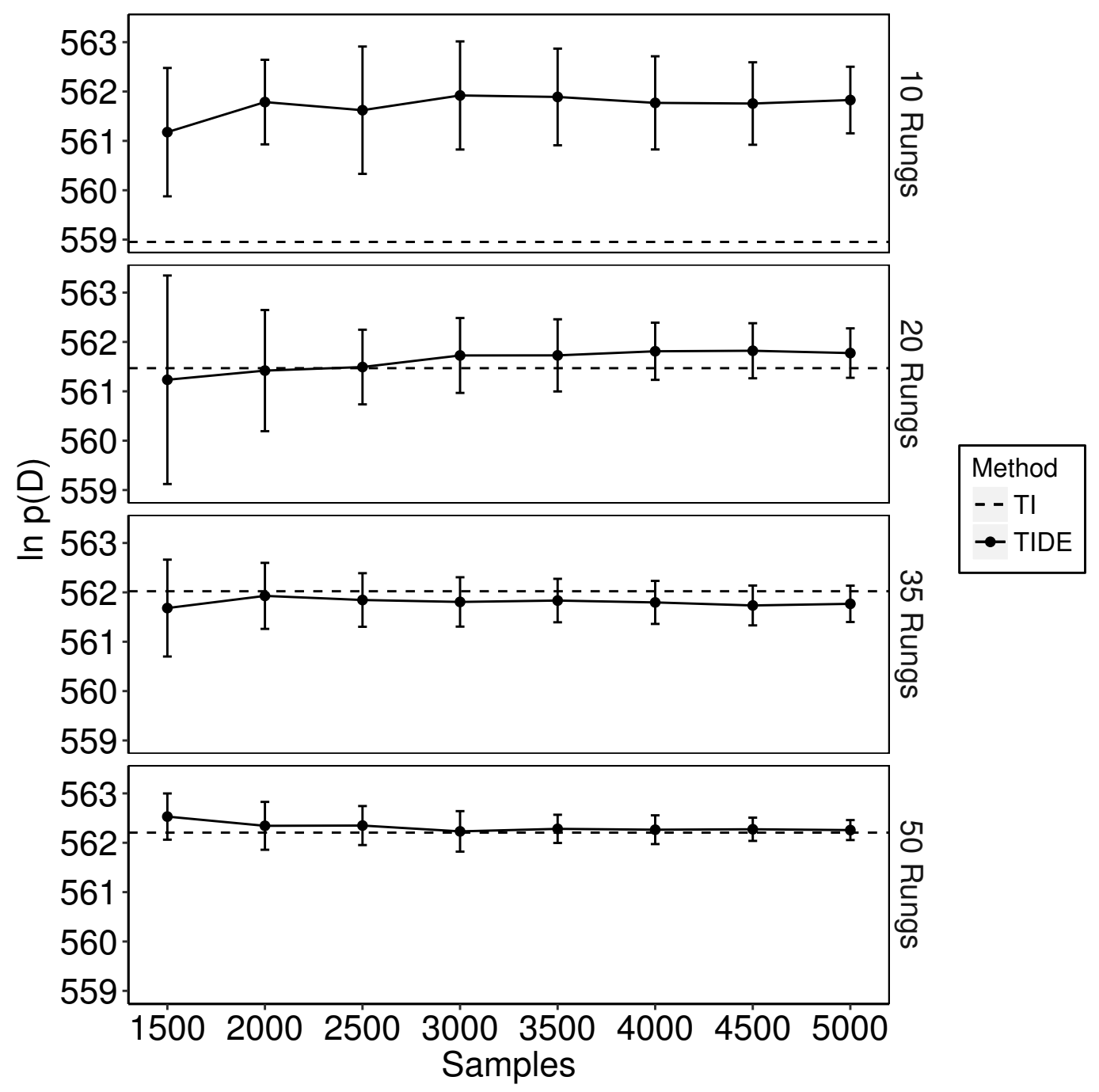

Figure 1. The estimated natural logarithm of the marginal likelihoods (y-axis) for the "simple" model across different numbers of samples (x-axis) and different numbers of temperatures used (different plots: known as "rungs"). The dashed lines display the values obtained through standard TI, and the solid lines display the values obtained through TIDE. Error bars are standard deviations based on 10 replications. 


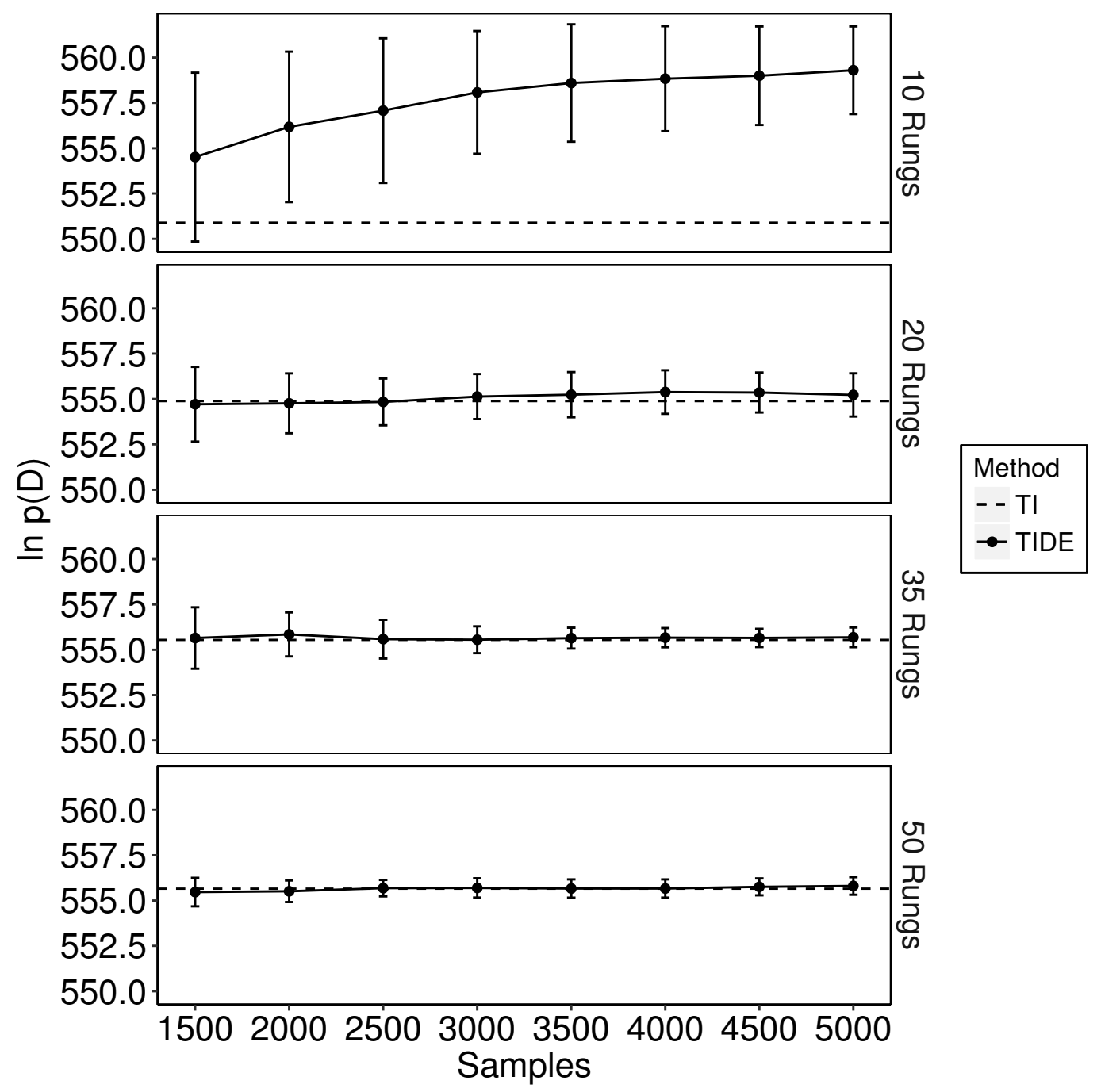

Figure 2. The estimated natural logarithm of the marginal likelihoods (y-axis) for the "complex" model across different numbers of samples (x-axis) and different numbers of temperatures used (different plots: known as "rungs"). The dashed lines display the values obtained through standard TI, and the solid lines display the values obtained through TIDE. 


\section{Hierarchical Example}

As with the individual subjects simulations, we defined both a "simple" and "complex" model. In addition, we defined two other models commonly of interest in applications of evidence accumulation models: a "drift-rate only" model, and a "threshold only" model. Lastly, in addition to defining a "simple" dataset, we also defined a "drift-rate" dataset, which had the same parameters varying across conditions as in the data-generating process of the "drift-rate only" model. The specific model definition for data generation and fitting can be found in the Appendix. For each model fit to each dataset, we used standard TI without random pairing (i.e., dependent sampling between individual and group level parameters), standard TI with random pairing (i.e., independent sampling between individual and group level parameters), TIDE (i.e., dependent sampling between individual and group level parameters), and TIDEz (i.e., independent sampling between individual and group level parameters).

The results of applying these methods can be seen in Figure 3 for the simple dataset, and Figure 4 for the drift-rate dataset. In each figure, the left panel displays the methods that use dependent sampling of individual and group-level parameters, and the right panel displays the methods that use independent sampling. Each method was run 10 independent times using 35 temperature rungs, with points representing means and error bars representing standard deviations. First, and perhaps most interestingly, the use of dependent or independent sampling has a large effect on the approximated log-marginal likelihood, with dependent sampling resulting in much larger log-marginal likelihoods. This suggests that the seemingly minor change to the dependency of the sampling can have a potentially large impact on the approximated log-marginal likelihood, meaning that these sampling assumption should be carefully considered, and not chosen arbitrarily.

Second, although all methods (when matched on the dependency assumption) ap- 
pear to fairly closely agree in most situations, there appears to be some large differences in the approximated log-marginal likelihood for standard TI and TIDE for each of the models in the simple data (Figure 3, left panel). Interestingly, the model orderings remain the same, but the TIDE marginal likelihoods appear to be some constant factor lower than those obtained from standard TI (i.e., about 30 on the log scale). However, this may not necessarily indicate an inaccuracy of TIDE, as potential sampling problems were the original reason for the switch from dependent sampling to independent sampling, meaning that either (or both) method(s) may be inaccurate with the dependency included. Overall though, TIDE and TIDEz appear to show good agreement with TI in many situations, despite TIDE/TIDEz having fewer samples per temperature than TI, suggesting that TIDE/TIDEz may be promising methods for efficiently approximating marginal likelihoods. However, we believe that the discrepancies observed here motivate future work with a large-scale comparison between TI, TIDE, and some of the other marginal likelihood approximation methods (e.g., bridge sampling; Gronau et al., 2017), using both dependence and independence assumptions about the individual and group level parameters, in order to assess the agreement between methods that are intended to approximate the same quantity. 


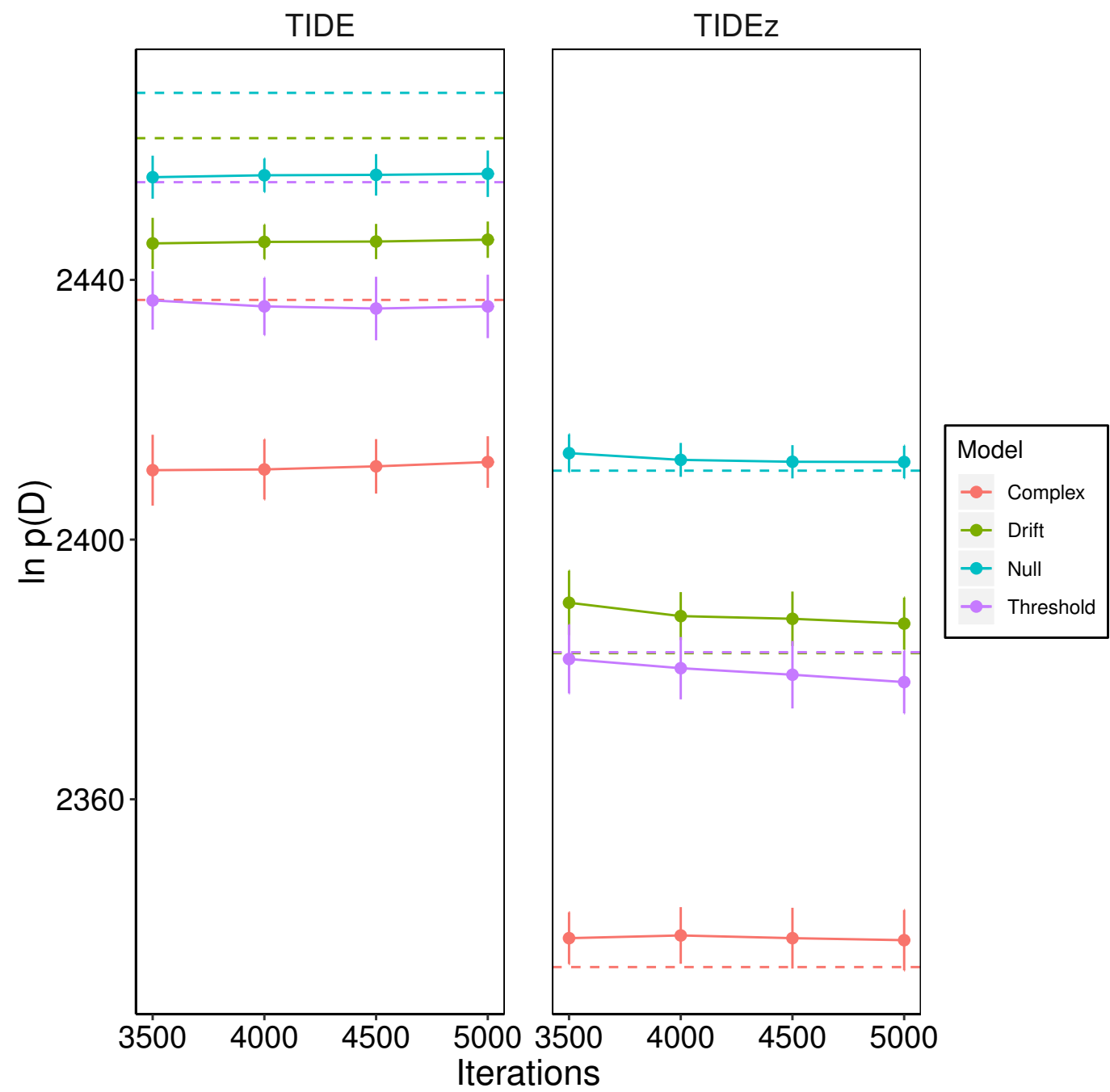

Figure 3. The estimated natural logarithm of the marginal likelihoods (y-axis) for each of the different models (different lines) across different numbers of samples (x-axis) on the "simple" dataset. The left panel displays TIDE, which uses dependent sampling between the individual-level and group-level parameters, whereas the right panel displays TIDEz, which uses independent sampling between the individual-level and group-level parameters. The dashed lines display the values obtained through standard TI, which differ between panels based on sampling dependency, the solid lines with circular points display the values obtained through TIDE/TIDEz. 


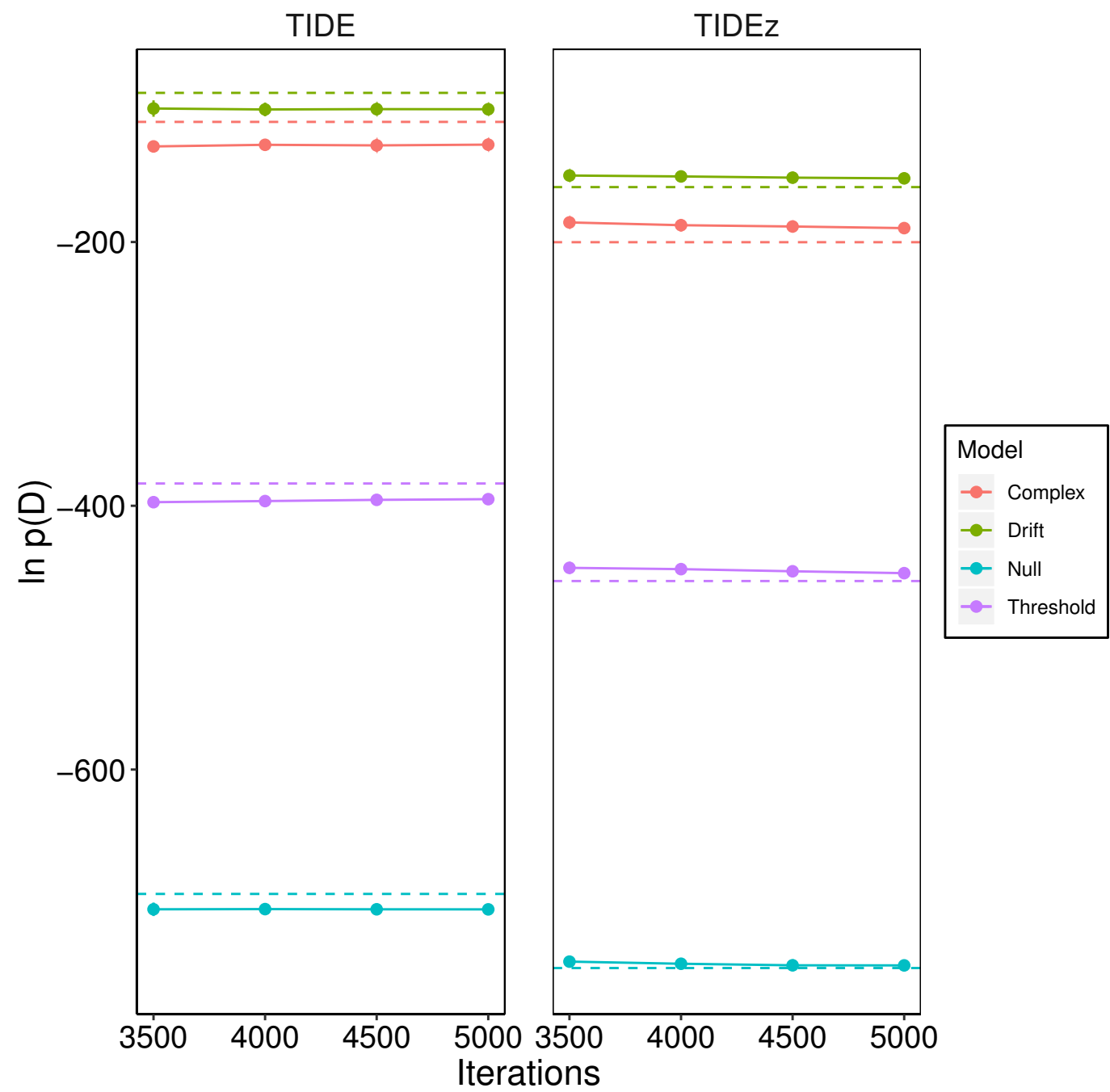

Figure 4. The estimated natural logarithm of the marginal likelihoods (y-axis) for each of the different models (different lines) across different numbers of samples (x-axis) on the "drift-rate" dataset. The left panel displays TIDE, which uses dependent sampling between the individuallevel and group-level parameters, whereas the right panel displays TIDEz, which uses independent sampling between the individual-level and group-level parameters. The dashed lines display the values obtained through standard TI, which differ between panels based on sampling dependency, the solid lines with circular points display the values obtained through TIDE/TIDEz. 


\section{Application to empirical data}

Although TIDE and TIDEz appear to produce sensible results in simulated environments where the generating process is known, the additional noise and uncertainty of empirical data can result in greater difficulties in selecting between competing models (Evans \& Brown, 2018). To see whether empirical data would prove problematic for TIDE/TIDEz, we applied the method to the data of Rae, Heathcote, Donkin, Averell, and Brown (2014), which have been used in the previous papers of Evans and Brown (2018) and Annis et al. (2018) as a benchmark. For brevity, we will only provide the essential details of the Rae et al. (2014) study here, though interested readers can see more in Rae et al. (2014), Evans and Brown (2018), or Annis et al. (2018).

In the study of Rae et al. (2014), each participant completed a perceptual decisionmaking task under two different sets of emphasis instructions: speed and accuracy. The key finding of the study was that both drift rate and threshold changed as a function of emphasis, as opposed to previous assumptions that emphasis only influenced threshold. Following Annis et al. (2018), we fit four models to these data: one that allow no parameters to vary over emphasis, one that allowed only drift rate to vary, one that allows only threshold to vary, and one that allowed both drift rate and threshold to vary. The exact model definitions are identical to those of (Annis et al., 2018). We fit each model using 35 parallel chains (i.e., 35 temperature rungs), with 5,000 samples per chain discarded as burn-in, and 3,000 samples per chain used to calculated the mean log-likelihood for each temperature.

The results of the fits can be seen in Figure 5 (TIDE) and Figure 6 (TIDEz). The $\mathrm{x}-$ axis displays different models, and the y-axis displays the estimated log-marginal likelihood, with larger numbers suggesting a better model. We ran 10 independent fitting routines for each model, with the column bars (standard TI) and circle (TIDE/TIDEz) on the graph 
represented the mean estimated marginal likelihood over these 10 fits, and error bars being omitted as the standard deviation in the estimate was smaller than the circle marker used to display the means. These results seem to indicate that both TIDE and TIDEz perform well when applied to empirical data: both methods shows little variability in the estimated log-marginal likelihood, and both select the drift rate and threshold model as the best model, with all models in the same ordering as standard TI. 


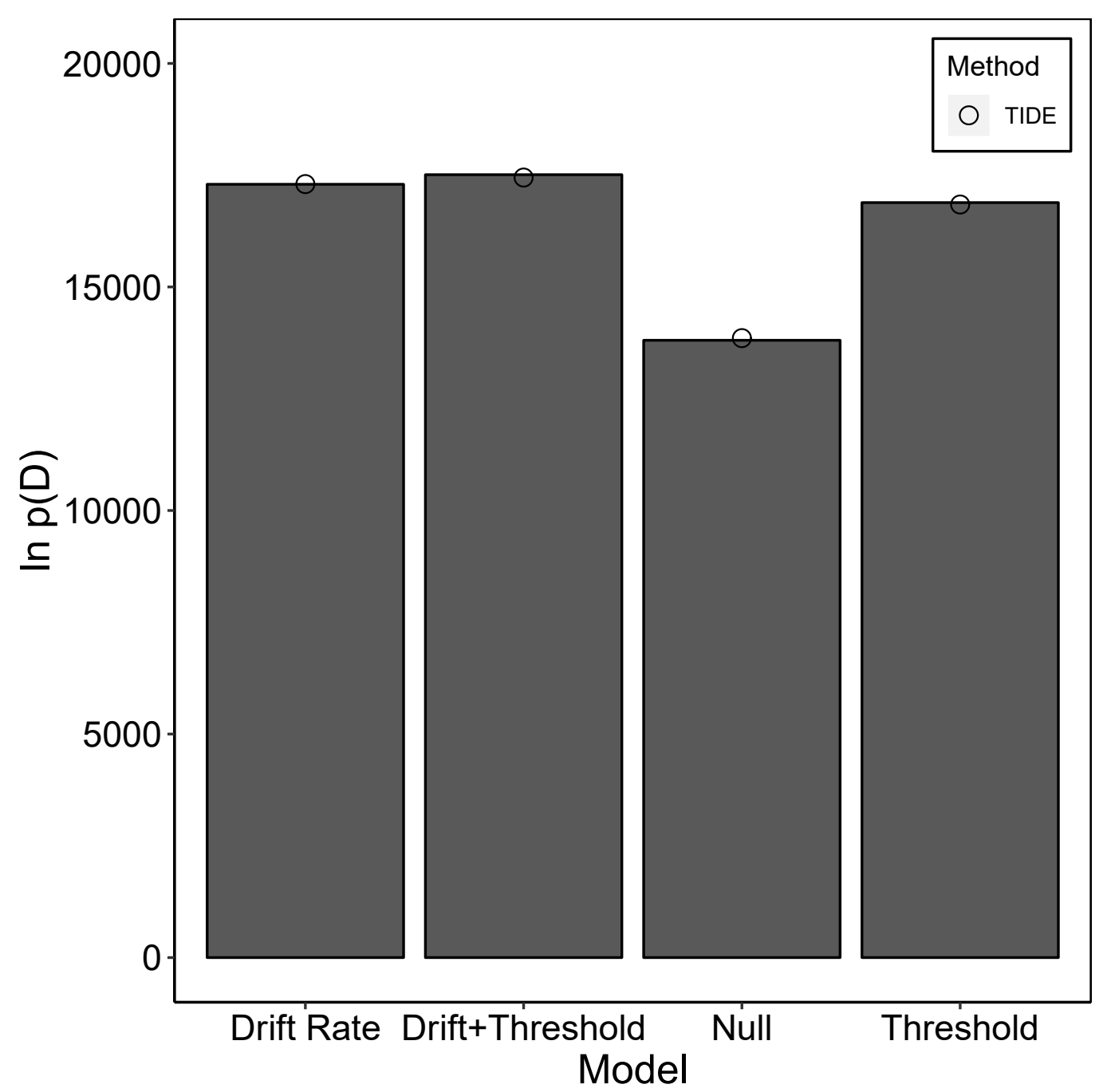

Figure 5. The estimated natural logarithm of the marginal likelihoods (y-axis) for each of the different models (x-axis) on the dataset of Rae et al. (2014). Column bars display the standard TI approximation with dependent sampling between individual and group level parameters, and circles display the TIDE approximation. Error bars for the TIDE approximation have not been included, as they were smaller that the circles used to represent the approximation. 


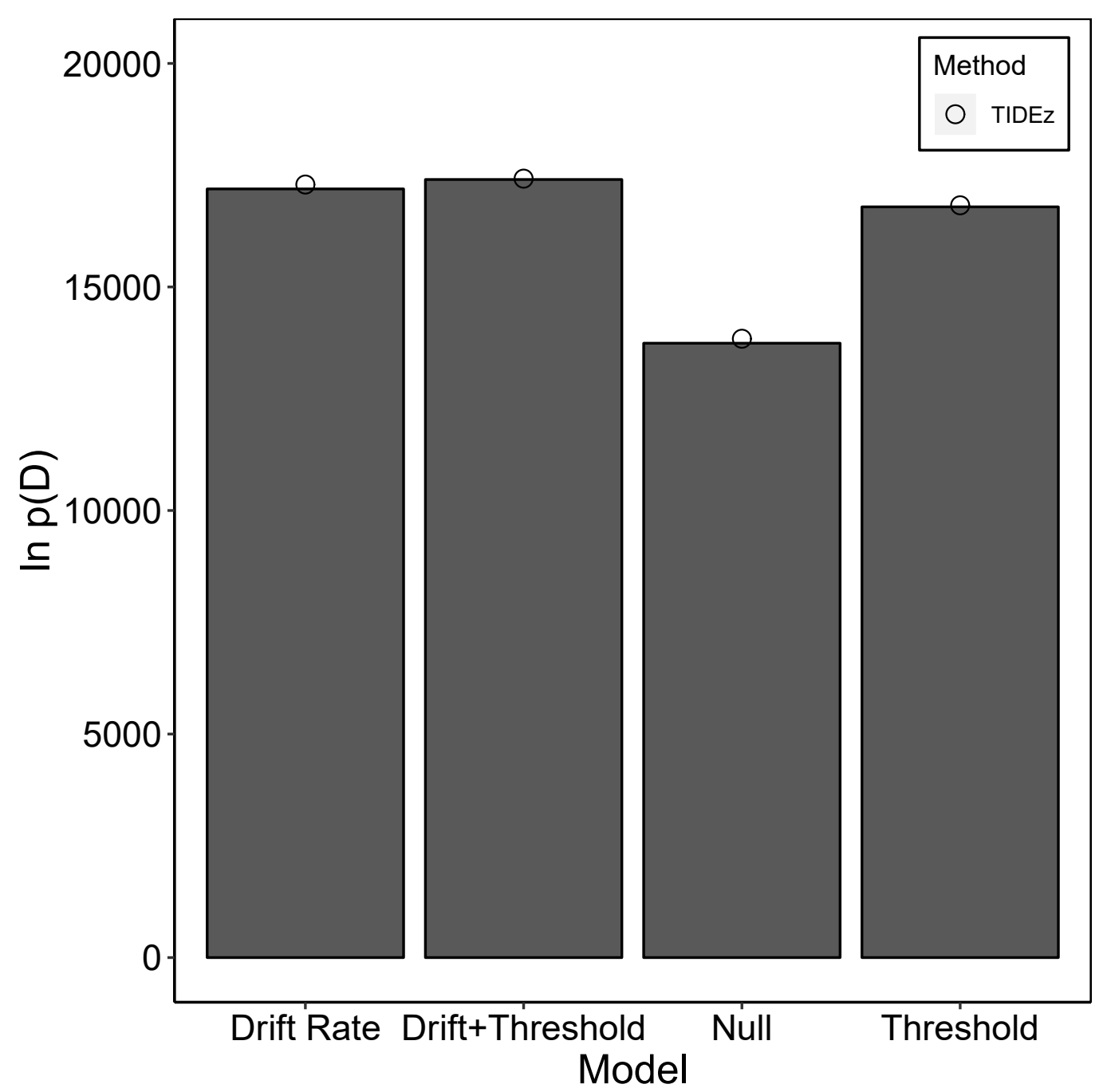

Figure 6. The estimated natural logarithm of the marginal likelihoods (y-axis) for each of the different models (x-axis) on the dataset of Rae et al. (2014). Column bars display the standard TI approximation with independent sampling between individual and group level parameters, and circles display the TIDEz approximation. Error bars for the TIDEz approximation have not been included, as they were smaller that the circles used to represent the approximation. 


\section{Discussion}

The aim of this article was to provide a simple, computationally efficient method of calculating Bayes factors for complex psychological models with correlated parameters. We proposed TIDE, an extension to TI that integrates the DE-MCMC method through the logic of Calderhead and Girolami's (2009) TI through population MCMC. As discussed earlier, TI requires many of MCMC runs over a set of power posteriors to obtain the marginal likelihood, whereas TIDE only requires a single MCMC run, which can increase the computational efficiency. We found that TIDE also closely matches the TI approximation of the log-marginal likelihood for when applied to the data of individual subjects using the LBA (Brown \& Heathcote, 2008). However, when applied to hierarchical models, the methods match somewhat less closely, and in one situation standard TI and TIDE show large differences, though by what appears to be some constant offset. We believe that TIDE provides an promising and simple to implement method of estimating the marginal likelihood of complex cognitive models, which will likely allow these approximations to be performed with minimal computational resources, such as personal computers. Our code for implementing TIDE in $R$ (Team et al., 2013) can be found at https://osf .io/ntmgw/, though note that the implementation of TIDE from a standard DE algorithm is extremely easy, and only requires assigning a temperature to each chain. We also include all simulated data sets used within this manuscript with the code, as a benchmark for those who wish to check their custom-written TIDE algorithms.

It is also important to note that DE-MCMC contains some unique limitations based on the crossover step used to generated proposals (ter Braak \& Vrugt, 2008), which may potentially be solved by TIDE. Specifically, as more chains arrive at the high-density areas of the posterior distribution over the course of sampling, the proposed jump steps more commonly become smaller. When the likelihood function of the model has many peaks and 
troughs, as is often the case in models with correlated parameters, it can be difficult, or impossible, for a proposal to be made that would result in the remaining chain(s) moving into the posterior region. This can result in a few chains getting "stuck" in regions outside the posterior, meaning that the sampled posterior distribution would be contaminated with some samples that are not truly from the posterior. These problems can be easily spotted through visual inspection of the chains, or standard convergence statistics (e.g., $\hat{R}$; see Gelman \& Rubin, 1992), and can often be overcome with techniques such as "migration" (attempting to exchange parameter values between chains; see Turner et al., 2013) or variable jump steps (ter Braak \& Vrugt, 2008). However, these solutions are not always effective (ter Braak \& Vrugt, 2008), and incorrect use of migration can result in convergence to a local maxima, or the sampling of an overly narrow posterior (i.e., not the true posterior distribution). Interestingly, TIDE also provides a potential solution to the "stuck" chain problem of DE-MCMC, as the interaction between temperatures in TIDE produce a natural variability in the size of jumps proposed with each chain estimating a different distribution. This eliminates the need for techniques like "migration" or variable jump steps, meaning that every proposal is based on the same algorithm, and therefore, implementation is more straight-forward. Indeed, we did not use migration for TIDE or TIDEz in any example within this article, and did not appear to encounter any problems with sampling from the correct posterior distributions. Therefore, TIDE may provide some attractive properties beyond efficiency.

Lastly, it should be made clear that there were several discrepancies, both minor and major, when marginal likelihoods were approximated for hierarchical models. Standard TI and TIDE did not show extremely close agreement in the hierarchical cases - in contrast to the assessment of individual subjects - and the use of dependent vs. independent sampling of individual and group-level parameters resulted in large differences in the approximated 
marginal likelihoods. Therefore, we believe that future research should aim to perform a detailed comparison between different methods of approximating marginal likelihoods for cognitive models (including other methods, such as bridge sampling; Gronau et al., 2017), as well as a more detailed assessment of whether sampling assumptions can make meaningful differences on inferences between models, and which assumptions seem most sensible. 


\section{References}

Akaike, H. (1974). A new look at the statistical model identification. Automatic Control, IEEE Transactions on, 19(6), 716-723.

Annis, J., Evans, N. J., Miller, B. J., \& Palmeri, T. J. (2018). Thermodynamic integration and steppingstone sampling methods for estimating bayes factors: A tutorial. Retrieved from psyarxiv.com/r8sgn.

Brown, S. D., \& Heathcote, A. (2008). The simplest complete model of choice response time: linear ballistic accumulation. Cognitive psychology, 57(3), 153-178.

Brown, S. D., Marley, A. A. J., Donkin, C., \& Heathcote, A. (2008). An integrated model of choices and response times in absolute identification. Psychological review, 115(2), 396.

Calderhead, B., \& Girolami, M. (2009). Estimating bayes factors via thermodynamic integration and population mcmc. Computational Statistics 83 Data Analysis, 53(12), 4028-4045.

Chib, S. (1995). Marginal likelihood from the gibbs output. Journal of the american statistical association, 90(432), 1313-1321.

Donkin, C., Brown, S. D., \& Heathcote, A. (2009). The overconstraint of response time models: Rethinking the scaling problem. Psychonomic Bulletin \& Review, 16(6), 1129-1135.

Evans, N. J., \& Brown, S. D. (2017). People adopt optimal policies in simple decision-making, after practice and guidance. Psychonomic Bulletin \&3 Review, 24 (2), 597-606.

Evans, N. J., \& Brown, S. D. (2018). Bayes factors for the linear ballistic accumulator model of decision-making. Behavior research methods, 50(2), 589-603.

Evans, N. J., Rae, B., Bushmakin, M., Rubin, M., \& Brown, S. D. (2017). Need for closure is associated with urgency in perceptual decision-making. Memory $\&$ Cognition, 1-13.

Evans, N. J., Steyvers, M., \& Brown, S. D. (2018). Modeling the covariance structure of complex datasets using cognitive models: An application to individual differences and the heritability of cognitive ability. Cognitive science.

Friel, N., \& Pettitt, A. N. (2008). Marginal likelihood estimation via power posteriors. Journal of the Royal Statistical Society: Series B (Statistical Methodology), 70(3), 589-607.

Friel, N., \& Wyse, J. (2012). Estimating the evidence-a review. Statistica Neerlandica, 66(3), 
$288-308$.

Gelman, A., \& Rubin, D. B. (1992). Inference from iterative simulation using multiple sequences. Statistical science, 457-472.

Gronau, Q. F., Sarafoglou, A., Matzke, D., Ly, A., Boehm, U., Marsman, M., ... Steingroever, H. (2017). A tutorial on bridge sampling. Journal of mathematical psychology, 81, 80-97.

Heathcote, A., Lin, Y.-S., Reynolds, A., Strickland, L., Gretton, M., \& Matzke, D. (2018). Dynamic models of choice. Behavior research methods, 1-25.

Ho, T. C., Yang, G., Wu, J., Cassey, P., Brown, S. D., Hoang, N., ... others (2014). Functional connectivity of negative emotional processing in adolescent depression. Journal of affective disorders, $155,65-74$

Kass, R. E., \& Raftery, A. E. (1995). Bayes factors. Journal of the american statistical association, 90(430), 773-795.

Lartillot, N., \& Philippe, H. (2006). Computing bayes factors using thermodynamic integration. Systematic biology, 55(2), 195-207.

Liu, P., Elshall, A. S., Ye, M., Beerli, P., Zeng, X., Lu, D., \& Tao, Y. (2016). Evaluating marginal likelihood with thermodynamic integration method and comparison with several other numerical methods. Water Resources Research, 52(2), 734-758.

Lodewyckx, T., Kim, W., Lee, M. D., Tuerlinckx, F., Kuppens, P., \& Wagenmakers, E.-J. (2011). A tutorial on bayes factor estimation with the product space method. Journal of Mathematical Psychology, 55(5), 331-347.

Myung, I. J. (2000). The importance of complexity in model selection. Journal of Mathematical Psychology, 44(1), 190-204.

Myung, I. J., \& Pitt, M. A. (1997). Applying occams razor in modeling cognition: A bayesian approach. Psychonomic Bulletin \& Review, 4 (1), 79-95.

Rae, B., Heathcote, A., Donkin, C., Averell, L., \& Brown, S. (2014). The hare and the tortoise: Emphasizing speed can change the evidence used to make decisions. Journal of Experimental Psychology: Learning, Memory, and Cognition, 40(5), 1226.

Ratcliff, R. (1978). A theory of memory retrieval. Psychological review, 85(2), 59.

Ratcliff, R., \& Rouder, J. N. (1998). Modeling response times for two-choice decisions. Psychological 
Science, 9(5), 347-356.

Roberts, S., \& Pashler, H. (2000). How persuasive is a good fit? a comment on theory testing. Psychological review, 107(2), 358.

Schwarz, G., et al. (1978). Estimating the dimension of a model. The annals of statistics, 6(2), $461-464$.

Shiffrin, R. M., Lee, M. D., Kim, W., \& Wagenmakers, E.-J. (2008). A survey of model evaluation approaches with a tutorial on hierarchical bayesian methods. Cognitive Science, 32(8), 12481284 .

Stone, M. (1960). Models for choice-reaction time. Psychometrika, 25(3), 251-260.

Team, R. C., et al. (2013). R: A language and environment for statistical computing.

Ter Braak, C. J. (2006). A markov chain monte carlo version of the genetic algorithm differential evolution: easy bayesian computing for real parameter spaces. Statistics and Computing, 16(3), 239-249.

ter Braak, C. J., \& Vrugt, J. A. (2008). Differential evolution markov chain with snooker updater and fewer chains. Statistics and Computing, 18(4), 435-446.

Tillman, G., Osth, A. F., van Ravenzwaaij, D., \& Heathcote, A. (2017). A diffusion decision model analysis of evidence variability in the lexical decision task. Psychonomic bulletin $\&$ review, $24(6), 1949-1956$.

Turner, B. M., Sederberg, P. B., Brown, S. D., \& Steyvers, M. (2013). A method for efficiently sampling from distributions with correlated dimensions. Psychological methods, 18(3), 368.

Wagenmakers, E.-J., Lodewyckx, T., Kuriyal, H., \& Grasman, R. (2010). Bayesian hypothesis testing for psychologists: A tutorial on the savage-dickey method. Cognitive psychology, $60(3), 158-189$.

Wiecki, T. V., Sofer, I., \& Frank, M. J. (2013). Hddm: hierarchical bayesian estimation of the drift-diffusion model in python. Frontiers in neuroinformatics, 7, 14.

Xie, W., Lewis, P. O., Fan, Y., Kuo, L., \& Chen, M.-H. (2010). Improving marginal likelihood estimation for bayesian phylogenetic model selection. Systematic biology, 60(2), 150-160. 


\section{Appendix A}

\section{Thermodynamic Integration Derivation}

Thermodynamic integration (TI; Friel \& Pettitt, 2008; Lartillot \& Philippe, 2006) represents the marginal likelihood as a one-dimensional integral, which can then be estimated using standard numerical integration techniques. In this section, we show the derivation for TI. To begin, the likelihood in the posterior is raised to a power, $t$. This leads to the power posterior:

$$
p(\boldsymbol{\theta} \mid \boldsymbol{D}, t)=\frac{p(\boldsymbol{D} \mid \boldsymbol{\theta})^{t} p(\boldsymbol{\theta})}{p(\boldsymbol{D} \mid t)}
$$

The power posterior has the following marginal likelihood, which we refer to as the power marginal likelihood:

$$
p(\boldsymbol{D} \mid t)=\int p(\boldsymbol{\theta} \mid \boldsymbol{D})^{t} p(\boldsymbol{\theta}) d \boldsymbol{\theta}, t \in[0,1]
$$

Given this formulation of the marginal likelihood, it is possible to write the log of the marginal likelihood as a difference between log-marginal likelihoods at $t=1$ and $t=$ 0, omitting the notation for the model:

$$
\ln p(D)=\ln p(D \mid t=1)-\ln p(D \mid t=0)
$$

where $p(D \mid t=0)$ is a prior distribution that integrates to unity (i.e., is proper) and therefore returns zero when the log is taken. We then introduce the following identity:

$$
\ln p(\boldsymbol{D} \mid t=1)-\ln p(\boldsymbol{D} \mid t=0)=\int_{0}^{1} \frac{d}{d t} \ln p(\boldsymbol{D} \mid t) d t
$$

Taking the derivative with respect to $t$ we find the following: 


$$
\begin{aligned}
\frac{d}{d t} \ln p(\boldsymbol{D} \mid t) & =\frac{1}{p(\boldsymbol{D} \mid t)} \frac{d}{d t} p(\boldsymbol{D} \mid t) \\
& =\frac{1}{p(\boldsymbol{D} \mid t)} \int p(\boldsymbol{D} \mid \boldsymbol{\theta})^{t} \ln p(\boldsymbol{D} \mid \boldsymbol{\theta}) p(\boldsymbol{\theta}) d \boldsymbol{\theta} \\
& =\int \frac{p(\boldsymbol{D} \mid \boldsymbol{\theta})^{t} p(\boldsymbol{\theta})}{p(\boldsymbol{D} \mid t)} \ln p(\boldsymbol{D} \mid \boldsymbol{\theta}) d \boldsymbol{\theta} \\
& =E_{\boldsymbol{\theta} \mid \boldsymbol{D}, t} \ln p(\boldsymbol{D} \mid \boldsymbol{\theta})
\end{aligned}
$$

Substituting this result into Equation A4 we see that the log-marginal likelihood is the integral of the expected posterior deviance from 0 to 1 with respect to the temperature, $t:$

$$
\ln p(\boldsymbol{D})=\int_{0}^{1} \mathbf{E}_{\boldsymbol{\theta} \mid \boldsymbol{D}, t} \ln p(\boldsymbol{D} \mid \boldsymbol{\theta}) d t
$$

This result indicates that the log-marginal likelihood can be expressed as a onedimensional integral, which can be approximated with standard numerical integration techniques.

\section{Hierarchical Setting}

The derivation for TI in the hierarchical setting is straightforward. The hierarchical structure assumed is given by:

$$
\begin{aligned}
\boldsymbol{D}_{p} & \sim p\left(\boldsymbol{D}_{p} \mid \boldsymbol{\theta}_{p}\right) \\
\boldsymbol{\theta}_{p} & \sim p\left(\boldsymbol{\theta}_{p} \mid \boldsymbol{\phi}\right) \\
\boldsymbol{\phi} & \sim p(\boldsymbol{\phi}),
\end{aligned}
$$


where $p$ indexes the participant, $\boldsymbol{\phi}$ are the group-level parameters, and $\boldsymbol{\theta}$ are the individuallevel parameters. The power posterior is given by an application of Bayes' rule:

$$
p(\boldsymbol{\theta}, \boldsymbol{\phi} \mid \boldsymbol{D}, t)=\frac{p(\boldsymbol{D} \mid \boldsymbol{\theta}, \boldsymbol{\phi})^{t} p(\boldsymbol{\theta}, \boldsymbol{\phi})}{p(\boldsymbol{D} \mid t)} .
$$

The structure of the hierarchical model is such that the data are conditionally independent of the group-level parameters. This allows us to write the power posterior as:

$$
p(\boldsymbol{\theta}, \boldsymbol{\phi} \mid \boldsymbol{D}, t)=\frac{p(\boldsymbol{D} \mid \boldsymbol{\theta}, \boldsymbol{\phi})^{t} p(\boldsymbol{\theta} \mid \boldsymbol{\phi}) p(\boldsymbol{\phi})}{p(\boldsymbol{D} \mid t)},
$$

where the power marginal likelihood is given by:

$$
p(\boldsymbol{D} \mid t)=\iint p(\boldsymbol{D} \mid \boldsymbol{\theta}, \boldsymbol{\phi})^{t} p(\boldsymbol{\theta} \mid \boldsymbol{\phi}) p(\boldsymbol{\phi}) d \boldsymbol{\theta} d \boldsymbol{\phi}
$$

Recall that TI relies on taking the log of the power marginal likelihood and finding its derivative:

$$
\frac{d}{d t} \ln p(\boldsymbol{D} \mid t)=\iint \frac{p(\boldsymbol{D} \mid \boldsymbol{\theta})^{t} p(\boldsymbol{\theta} \mid \boldsymbol{\phi}) p(\boldsymbol{\phi})}{p(\boldsymbol{D} \mid t)} \ln p(\boldsymbol{D} \mid \boldsymbol{\theta}) d \boldsymbol{\theta} d \boldsymbol{\phi}
$$

This leads to the following expectation:

$$
\frac{d}{d t} \ln p(\boldsymbol{D} \mid t)=\mathbf{E}_{\boldsymbol{\theta}, \boldsymbol{\phi} \mid \boldsymbol{D}, t} \ln p(\boldsymbol{D} \mid \boldsymbol{\theta})
$$

Substituting this result into Equation A4 we have the following one-dimensional integral representation of the marginal likelihood:

$$
\ln p(\boldsymbol{D})=\int_{0}^{1} \mathbf{E}_{\boldsymbol{\theta}, \boldsymbol{\phi} \mid \boldsymbol{D}, t} \ln p(\boldsymbol{D} \mid \boldsymbol{\theta}) d t
$$


This implies the computation of the TI marginal likelihood estimate involves sampling from the joint power posterior, $p(\boldsymbol{\theta}, \boldsymbol{\phi} \mid \boldsymbol{D}, t)$, and then using the individual-level samples, $\boldsymbol{\theta}_{i}$, to compute the average log-likelihood.

\section{Appendix B}

\section{Model definitions}

Individual subjects analysis

The data for this "simple" dataset were generated with the following parameter values:

$$
\begin{array}{r}
v . c=3.5 \\
v . e=1 \\
s . c=1 \\
s . e=1 \\
b-A=0.4 \\
A=1 \\
t 0=0.3
\end{array}
$$

where.$c$ and.$e$ refer to the accumulators for correct and error responses, respectively.

The "simple" model had the following prior distributions: 


$$
\begin{aligned}
& v . c \sim N_{+}(3,3) \\
& v . e \sim N_{+}(1,1) \\
& s . e \sim N_{+}(1,1) \\
& b-A \sim N_{+}(0.4,0.4) \\
& A \sim N_{+}(1,1) \\
& t 0 \sim N_{+}(0.3,0.3)
\end{aligned}
$$

where s.c was fixed to 1 to satisfy a scaling property in the model (Donkin, Brown, \& Heathcote, 2009), and $N_{+}$refers to the normal distribution truncated to only positive values. The "complex" model had the following prior distributions:

$$
\begin{array}{r}
v . c_{j} \sim N_{+}(3,3) \\
v . e \sim N_{+}(1,1) \\
s . e \sim N_{+}(1,1) \\
b_{j}-A \sim N_{+}(0.4,0.4) \\
A \sim N_{+}(1,1) \\
t 0_{j} \sim N_{+}(0.3,0.3)
\end{array}
$$

where $j$ indexes the condition. 


\section{Hierarchical subjects analysis}

The data for the hierarchical analysis were generated using distributions of parameter values, where each of the 10 simulated subjects had parameter values randomly drawn from these distributions. For the "simple" dataset, the following distributions were used:

$$
\begin{array}{r}
v . c_{i} \sim N_{+}(3.5,0.35) \\
v . e_{i} \sim N_{+}(1,0.1) \\
s . e_{i} \sim N_{+}(1,01) \\
b_{i}-A_{i} \sim N_{+}(0.4,0.04) \\
A_{i} \sim N_{+}(1,01) \\
t 0_{i} \sim N_{+}(0.3,0.03)
\end{array}
$$

where $i$ indexes the participant, and for the "drift-only" dataset, the following distributions were used:

$$
\begin{aligned}
& v . c_{i, \text { cond } 1} \sim N_{+}(4,0.4) \\
& v . c_{i, \text { cond } 2} \sim N_{+}(3,0.3) \\
& v . e_{i} \sim N_{+}(1,0.1) \\
& s . e_{i} \sim N_{+}(1,0.1) \\
& b_{i}-A_{i} \sim N_{+}(0.4,0.04) \\
& A_{i} \sim N_{+}(1,0.1) \\
& t 0_{i} \sim N_{+}(0.3,0.03)
\end{aligned}
$$


where cond 1 and cond2 are the two "within-subjects" conditions.

All models had the following prior distributions in common:

$$
\begin{array}{r}
v . e_{i} \sim N_{+}\left(\mu_{v . e}, \sigma_{v . e}\right) \\
s . e_{i} \sim N_{+}\left(\mu_{s . e}, \sigma_{s . e}\right) \\
A_{i} \sim N_{+}\left(\mu_{A}, \sigma_{A}\right) \\
\mu_{v . e}, \mu_{s . e}, \mu_{A} \sim N_{+}(1,1) \\
\sigma_{v . e}, \sigma_{s . e}, \sigma_{A} \sim N_{+}(1,1)
\end{array}
$$

the "simple" model had the following prior distributions:

$$
\begin{array}{r}
v . c_{i} \sim N_{+}\left(\mu_{v . c}, \sigma_{v . c}\right) \\
t 0_{i} \sim N_{+}\left(\mu_{t 0}, \sigma_{t 0}\right) \\
b_{i}-A_{i} \sim N_{+}\left(\mu_{b}, \sigma_{b}\right) \\
\mu_{v . c}, \sigma_{v . c} \sim N_{+}(3,3) \\
\mu_{t 0}, \sigma_{t 0} \sim N_{+}(0.3,0.3) \\
\mu_{b}, \sigma_{b} \sim N_{+}(0.4,0.4)
\end{array}
$$

the "drift-rate only" model had the following prior distributions: 


$$
\begin{array}{r}
v . c_{i, j} \sim N_{+}\left(\mu_{v . c_{j}}, \sigma_{v . c_{j}}\right) \\
t 0_{i} \sim N_{+}\left(\mu_{t 0}, \sigma_{t 0}\right) \\
b_{i}-A_{i} \sim N_{+}\left(\mu_{b}, \sigma_{b}\right) \\
\mu_{v . c_{j}}, \sigma_{v . c_{j}} \sim N_{+}(3,3) \\
\mu_{t 0}, \sigma_{t 0} \sim N_{+}(0.3,0.3) \\
\mu_{b}, \sigma_{b} \sim N_{+}(0.4,0.4)
\end{array}
$$

where $j$ indexes the condition, the "threshold only" model had the following prior distributions:

$$
\begin{aligned}
v . c_{i} & \sim N_{+}\left(\mu_{v . c}, \sigma_{v . c}\right) \\
t 0_{i} & \sim N_{+}\left(\mu_{t 0}, \sigma_{t 0}\right) \\
b_{i, j}-A_{i} & \sim N_{+}\left(\mu_{b_{j}}, \sigma_{b_{j}}\right) \\
\mu_{v . c}, \sigma_{v . c} & \sim N_{+}(3,3) \\
\mu_{t 0}, \sigma_{t 0} & \sim N_{+}(0.3,0.3) \\
\mu_{b_{j}}, \sigma_{b_{j}} & \sim N_{+}(0.4,0.4)
\end{aligned}
$$

and the "complex" model had the following prior distributions: 


$$
\begin{array}{r}
v . c_{i, j} \sim N_{+}\left(\mu_{v . c_{j}}, \sigma_{v . c_{j}}\right) \\
t 0_{i, j} \sim N_{+}\left(\mu_{t 0_{j}}, \sigma_{t 0_{j}}\right) \\
b_{i, j}-A_{i} \sim N_{+}\left(\mu_{b_{j}}, \sigma_{b_{j}}\right) \\
\mu_{v . c_{j}}, \sigma_{v . c_{j}} \sim N_{+}(3,3) \\
\mu_{t 0_{j}}, \sigma_{t 0_{j}} \sim N_{+}(0.3,0.3) \\
\mu_{b}, \sigma_{b} \sim N_{+}(0.4,0.4)
\end{array}
$$

\section{Appendix C}

Theoretical comparison of computational workloads of TIDE and

In this section we compare, in a theoretical manner, the computational workloads of TIDE and standard TI. The key advantage of TIDE over standard TI is that it only requires a single chain per power posterior.

When assuming that TI and TIDE are implemented with identical MCMC samplers (i.e., DE-MCMC) where each sample takes an identical time (on average) to evaluate, and only a single computational core is available (i.e., no ability for multi-core parallelization), efficiency depends on only the number of samples that need to be taken for each method, and one evaluates the following argument:

$$
\frac{\left[B_{t i}+S_{t i}\right] \times C_{t i} \times T_{t i}}{\left[B_{\text {tide }}+S_{t i d e}\right] \times C_{t i d e} \times T_{t i d e}}>Y
$$

where $B$ is the number of burn-in samples per chain, $C$ is the number of chains per temperature, $T$ is the number of temperatures used, and $S$ is the number of samples 
required from the posterior per chain to create the integration curve. When the argument is true, then TIDE is more efficient than TI. In the case of TIDE, the method only requires one chain per temperature (i.e., $C_{t i d e}=1$ ), meaning that Equation $\mathrm{C} 1$ can be simplified to:

$$
\frac{\left[B_{t i}+S_{t i}\right] \times C_{t i} \times T_{t i}}{\left[B_{\text {tide }}+S_{\text {tide }}\right] \times T_{\text {tide }}}>Y
$$

When assuming that TI and TIDE are implemented with an equal number of temperatures, Equation C2 can be simplified again:

$$
\frac{\left[B_{t i}+S_{t i}\right] \times C_{t i}}{\left[B_{\text {tide }}+S_{\text {tide }}\right]}>Y
$$

which essentially means that whenever the total number of samples in the TI algorithm (i.e., $\left.B_{t i}+S_{t i}\right)$ is greater than the ratio of the total number of samples in the TIDE algorithm to the number of chains in the TI algorithm (i.e., $\frac{B_{t i d e}+S_{t i d e}}{C_{t i}}$ ), then TIDE will be more efficient than TI. Importantly, the number of chains for the standard DE-MCMC algorithm (used in TI for this situation) is commonly set to $3 k$, where $k$ is the number of free parameters, meaning that TIDE will become increasingly efficient compared to TI when the dimensionality of the model increases.

Introducing the potential for multiple processing cores, and therefore, cross-core parallelization, makes the efficiency criterion somewhat more complicated. In cases where the number of computational cores is less than or equal to the number of temperatures used, and the number of temperatures can be equally divided into computational cores (i.e., no remainder from the equation $\frac{T_{t i}}{n C P U}$, where $n C P U$ is the number of computing cores), TI provides the ability for completely independent parallelization, where parallelization can be used without any cost in efficiency. Although TIDE can be parallelized across chains 
within each iteration of the sampling algorithm, this type of parallelization still has some level of dependency (i.e., only one iteration can be done in parallel at a time), meaning that there is some cost in efficiency when using multiple cores for TIDE, which will differ from situation to situation. This can be reflected by re-writing Equation C3 as:

$$
\frac{\left(\left[B_{t i}+S_{t i}\right] \times C_{t i}\right) / n C P U}{\left(\left[B_{t i d e}+S_{t i d e}\right]\right) /\left(n C P U \times E_{\text {tide }}\right)}>Y
$$

where $E$ is a coefficient for how imperfect the TIDE parallelization is, with 0 reflecting no benefit of parallelization, and 1 reflecting perfect parallelization. Importantly, when $E_{t i d e}$ is small and $n C P U$ is large, TIDE can be less efficient than TI, meaning that in situations where multiple cores are available these factors should be considered.

Lastly, using a sampler other than DE-MCMC for the TI estimate also makes the efficiency criterion somewhat more complicated. Importantly, we can no longer assume that samples take the same amount of time to generate, meaning that efficiency can not be purely expressed in terms of the number of samples required to approximate the integration curve. This can be reflected by re-writing Equation C3 as:

$$
\frac{\left[B_{t i}+S_{t i}\right] \times C_{t i} \times t_{t i}}{\left[B_{t i d e}+S_{t i d e}\right] \times t_{t i d e}}>Y
$$

where $t$ is the average time taken to generate a sample in each of the sampling algorithms. Importantly, this means that the efficiency of TI can be increased by using a sampler that either 1) decreases the number of chains required without proportional increases to the time per sample, or 2) decreases the time per sample without proportion increases to the number of chains required. 